\title{
User Experience Measurement for Senior Friendly Product
}

\author{
Kwang Tae Jung ${ }^{1}$, Keyoung Jin Chun ${ }^{2}$, Byeong Hee Won ${ }^{2}$ \\ ${ }^{1}$ Korea University of Technology and Education, Department of Industrial Design Engineering, 1600 Chungjeolno, \\ Byeongchun, Cheonan, 330-708 \\ ${ }^{2}$ Korea Institute of Industrial Technology, Smart Welfare Technology R\&D Group
}

\begin{abstract}
Objective: This study introduces user experience measurement and its application for senior friendly products. Background: Measuring user experience for senior friendly product is very important for its design, but the measurement is not easy. Method: This study was focused on case studies for user experience measurement and its application for senior friendly product. Mobile patient lift and four wheeled walker that assist the old's mobility were studied. User experience was measured through interview and observation method. Result: From measuring the user experience, some usability issues were identified for the products. A method to apply QFD(quality function deployment) in design process was proposed. A design prototype was also developed considering user experience. Conclusion: User experience measurement for senior friendly products is key process for user centered design. Application: The method and result of this study can be applied to user experience measurement and design of senior friendly product.
\end{abstract}

Keywords: User experience, Mobile patient lift, Four wheeled walker, QFD, Design prototype

\section{Introduction}

고령자 인구는 평균 수명의 증가와 함께 빠르게 증가하고 있다. 우리나라는 2000년도에 고령화 사회(고령자 인구의 비율이 $70 \%$ 이상인 경우)로 진입하였고, 2018년에 고령사 회(고령자 인구의 비율이 $14 \%$ 이상인 경우)에 진입할 것으 로 예상되고, 2026년에는 초고령 사회(고령자 비율이 $20 \%$ 이상인 경우)에 진입할 것으로 예상되고 있다. 이러한 예측 을 볼 때, 우리나라는 고령화 사회에서 고령사회로 진입하는 데 단지 18 년의 기간이 걸릴 것으로 예상된다. Table 1 은 세계 주요국가의 고령화 사회, 고령사회, 초고령 사회의 진 입연도이다. Table 1에서 볼 수 있는 바와 같이 우리나라는 고령화 사회에서 고령사회로 진입하는데 18 년이 걸릴 것으 로 예상되지만, 미국은 72 년, 일본은 25 년, 독일은 40 년,
프랑스는 115 년이 걸렸다. 이러한 사실로 부터 우리나라의 고령화 속도가 얼마나 빨리 진행되고 있는지를 알 수 있다. 이러한 사실은 고령자를 위한 복지나 제품의 개발 등에 있어 여러 가지 측면에서 문제점을 초래할 수 있기 때문에, 다양 한 측면에서의 준비와 연구가 요구되고 있다.

일반적으로 고령자는 세 그룹; 완전히 의존적인(fully dependent) 고령자 그룹, 완전히 독립적인 (fully independent) 고령자 그룹, 그리고 부분적으로 의존적인(partially dependent person)으로 나눌 수 있다.

완전히 의존적인 고령자 그룹은 생활을 위해 다른 사람의 도움이 필수적이다. 반면 완전히 독립적인 고령자 그룹은 생 활을 위해 다른 사람의 도움을 별로 필요로 하지 않는다. 부 분적으로 의존적인 고령자들은 일상생활을 위해 다른 사람 의 도움을 필요로 하는 경우가 발생한다(Waters, 2011). 어 떠한 경우이든, 고령자의 이동(mobility)은 신체적 재활과

Corresponding Author: Kwang Tae Jung. Koreatech, Department of Industrial Design Engineering 1600 Chungjeolno, Byeongchun, Cheonan, $330-708$. Mobile: +82-10-8838-9306, E-mail: ktjung@koreatech.ac.kr Copyright@2013 by Ergonomics Society of Korea(pISSN:1229-1684 eISSN:2093-8462). All right reserved.

(c) This is an open-access article distributed under the terms of the Creative Commons Attribution Non-Commercial License(http://creativecommons.org/licenses/by-nc/3.0/), which permits unrestricted non-commercial use, distribution, and reproduction in any medium, provided the original work is properly cited. http://www.esk.or.kr 
Table 1. Aging speed in major countries

\begin{tabular}{c|c|c|c}
\hline & $\begin{array}{c}\text { Ageing society } \\
(7 \%)\end{array}$ & $\begin{array}{c}\text { Aged society } \\
(14 \%)\end{array}$ & $\begin{array}{c}\text { Super aged } \\
(20 \%)\end{array}$ \\
\hline Korea & 2000 & 2018 & 2026 \\
\hline US & 1942 & 2014 & 2030 \\
\hline Japan & 1970 & 1994 & 2006 \\
\hline Germany & 1932 & 1972 & 2010 \\
\hline France & 1864 & 1979 & 2019 \\
\hline
\end{tabular}

일생생활을 위해 필수적인 요소이다. (Ryu et al., 2004).

완전히 의존적인 고령자들의 이동을 위해 리프트는 가장 대표적인 고령친화제품 중의 하나이다. 리프트는 휠체어에서 침대로, 또는 침대에서 휠체어로 고령자를 이동시키는데 가 장 일반적으로 활용된다(Simonton, 1999). 또한 완전히 독 립적인 고령자들의 이동을 위한 가장 일반적인 고령친화제 품 중의 하나는 보행보조차(walker)이다. 제품 안전 사고의 분석 결과를 보면, 사고 유형별로 볼 때 추락, 넘어짐, 미끄 러짐이 $55.3 \%$, 충돌, 충격이 $7.5 \%$, 물체에 베이거나 찢어 지는 사고가 $4.5 \%$, 눌림, 끼임이 $4.0 \%$ 의 순이었는데(Korea Consumer Agency, 2007), 이를 볼 때 낙상 관련 사고가 무려 절반 이상을 차지하고 있는 것을 알 수 있다. 보행보조 차는 고령자의 이동과정에서 발생하는 낙상을 방지할 수 있 는 가장 대표적인 제품이다.

고령자를 위한 제품의 개발에 있어 제품의 사용성과 안전 성은 가장 중요하게 고려되어야 하는 요소이다. 고령친화제 품의 사용성과 안전성을 보장하기 위해 필요한 것이 해당 제품들에 대한 사용자들의 경험을 측정하여 디자인에 반영 하는 것이다. 본 연구에서는 고령친화제품에 대한 사용자 경 험 측정의 사례로, 고령자의 이동을 위해 대표적으로 사용되 는 이동리프트와 보행보조차를 대상으로 연구를 진행하였고, $\mathrm{QFD}$ 를 활용하여 측정된 사용자 경험을 디자인과정에서 반 영할 수 있는 방법을 제시하였으며, 보행보조차에 대해서는 사용자 경험을 고려한 디자인 프로토타입을 개발하였다.

\section{User Experience Measurement for Mobile Patient Lift}

최근 헬스케어 산업 분야에서는 환자를 케어하는 작업에 서 요구되는 요양보호사의 근골격질환 예방과 관련된 제품 의 개발에 많은 관심을 두고 있다(Waters, 2011). 그러한 측면에서 환자용 이동리프트 시장은 점차적으로 확대되고 있는데, 우리나라의 경우 일본을 포함한 선진국에 비해서는
그 규모가 아직도 작지만, 2008년 도입된 장기요양보험으로 인하여 시장 규모가 점차적으로 커지고 있는 추세에 있다 (Table 2).

Table 2. Market size of patient lift

(amount unit: \$1,000,000)

\begin{tabular}{c|c|c|c|c|c|c}
\hline \multirow{2}{*}{ Year } & \multicolumn{2}{|c|}{ World } & \multicolumn{2}{c|}{ Japan } & \multicolumn{2}{c}{ Korea } \\
\cline { 2 - 7 } & Quantity & Amount & Quantity & Amount & Quantity & Amount \\
\hline 2007 & 25,000 & 125.0 & 5,000 & 25.0 & 300 & 1.5 \\
\hline 2008 & 26,000 & 133.8 & 5,400 & 27.0 & 500 & 2.3 \\
\hline 2009 & 28,600 & 143.1 & 5,800 & 29.0 & 500 & 2.5 \\
\hline 2010 & 30,600 & 153.1 & 6,300 & 31.5 & 600 & 2.8 \\
\hline 2011 & 32,800 & 163.8 & 6,800 & 34.0 & 600 & 3.1 \\
\hline 2015 & 43,000 & 214.8 & 9,300 & 46.3 & 900 & 4.5 \\
\hline $\begin{array}{c}\text { Average } \\
\text { annual } \\
\text { growth } \\
\text { rate }\end{array}$ & \multicolumn{2}{|c|}{$7 \%$} & \multicolumn{2}{c}{$8 \%$} & \multicolumn{2}{c}{$10 \%$} \\
\hline
\end{tabular}

(Ministry of Health and Welfare in Korea, 2006)

미국 노동통계국(US Bureau of Labor Statistics)에 따 르면, 비보행 환자의 2 명 중 1 명이 이동 중에 떨어진 경험 이 있고, 3 명의 요양보호사 중 1 명이 환자의 이동 중 상해를 입은 경험이 있다고 보고되어 있다(OSHA, 2003). 특히 많 은 사고가 환자를 들고 내리는 과정에서 발생되는데, 이와 같은 작업에서 요양보호사의 신체적인 부담을 줄여 주는 것 이 이동리프트다.

따라서 환자를 이동시키는 작업에서의 요양보호사의 상해 비율이 높게 나타나고, 이동리프트에 대한 수요가 증가하는 시점에서, 이동리프트에 대한 사용자 경험을 측정하고, 디자 인에 반영함으로써 이동리프트 사용자들의 사용편의성과 안 전성을 제고하는 것이 필요하다.

\subsection{Qualitative user experience measurement}

본 연구에서는 서울시 용산구에 있는 '영락애니아의집'을 방문하여 이동리프트(바닥 주행 리프트)를 사용하고 있는 요양보호사 5명을 대상으로 인터뷰를 시행하였으며, 사용자 관찰 방법도 병행하여 이동리프트에 대한 사용자 경험을 측 정하였다. 원활하고 효과적인 진행을 위하여 인터뷰 항목을 미리 작성하여 심층적인 인터뷰를 진행하였다.

이동리프트에 대한 사용자 경험을 측정하기 위하여 조사 된 항목은 사용 용도 및 용도별 경험, 환자 및 요양보호사 특성과의 적합성, 실내 환경과의 적합성, 보관 시 문제점, 사 용과정에 관한 경험, 과거 사고 경험 및 원인, 사용편의성 및 
디자인에 관한 경험 등을 포함하였다.

요양보호사를 대상으로 한 인터뷰를 통하여 얻어진 디자 인 개선을 필요로 하는 사용자 경험들을 정리한 결과는 다 음과 같다.

- 신체지지 기구의 승, 하강 시 속도가 너무 느리다.

- 신체지지 기구(슬링)에 사용자가 앉았을 때 머리가 뒤 로 젖혀진다.

- 리프트 이동 시 신체지지 기구(슬링)가 움직여 사용자가 프레임에 자주 부딪친다.

-리프트 이동 시 움직이는 슬링을 붙잡기 위하여 두 명의 요양보호사가 필요하다.

-리프트 보관 시 공간을 많이 차지한다.

- 바퀴를 지지하는 프레임 부분에 요양보호사 및 주변 사 람들의 발이 부딪히는 사고가 자주 발생한다.

- (미숙련자일 경우)사용 시 시간이 오래 걸려 사용 빈도 가 매우 낮다.

- 요양보호사들이 슬링의 사용법을 제대로 알고 있지 않다. - 슬링의 사용법을 알지 못하여 휠체어에서 리프트 이동 시 어려움을 겪으며, 안전 사고 경험이 있다.

- 휠체어에서 슬링을 빼낼 때 슬링에 붙어있는 고리로 인 하여 사용자가 다치는 경우가 있다.

- 형태가 육중해 보이고 미적으로 만족스럽지 못하다.

또한 이동리프트 사용과정에 대한 사용자 관찰을 수행하 였는데, 이동리프트는 주로 목욕 시 많이 사용하여서 환자를 샤워 트롤리로 옮기는 과정을 중심으로 관찰을 진행하였다. 관찰에 의한 사용자 분석은 비디오카메라로 녹화하여 사후 분석하는 방법으로 진행하였다. 이동리프트 사용 시 동작은 유사한 동작끼리 그룹화하여 단계를 구분한 후 관찰 시 발견 된 문제점을 도출하였다(Table 3).

본 연구에서는 이동리프트에 대한 사용자 인터뷰와 관찰

Table 3. User experience measurement through observation

\begin{tabular}{c|l}
\hline Step & \multicolumn{1}{c}{ Problems } \\
\hline $\begin{array}{c}\text { Transferring a patient } \\
\text { to/from sling }\end{array}$ & $\begin{array}{l}\text { - Patient's postural variation for balancing } \\
\text { position. }\end{array}$ \\
\hline $\begin{array}{c}\text { Raising and lowering } \\
\text { a patient }\end{array}$ & $\begin{array}{l}\text { - A patient's head sinks back while raising. } \\
\text { - Raising and lowering speed is slow. } \\
\text { continuously swings while raising and } \\
\text { lowering. }\end{array}$ \\
\hline Lift movement & $\begin{array}{l}\text { - Knocking user's head against lift frame. } \\
\text { - Hard to move a lift by one helper. }\end{array}$ \\
\hline Storage & - Taking up too much space. \\
\hline
\end{tabular}

을 통하여 얻어진 사용자 경험 측정 결과를 토대로 다음의 9 가지 디자인과 관련한 이슈를 도출하였다.

- 환자를 올리고 내리는 과정이 쉬울 것

- 이동리프트를 이동하는 것이 쉬울 것

- 안전하게 이동할 수 있을 것

- 요양보호사의 상해를 방지할 수 있을 것

- 환자를 옮기는 동안 신체를 안정시킬 것

- 사용 환경에 적합할 것

• 슬링이 환자에게 잘 맞을 것

- 만족스러운 형태를 가질 것

- 브레이크 조절이 용이할 것

\subsection{Design direction through QFD}

사용자 경험을 고려한 디자인 방향을 설정하기 위하여, QFD(Quality Function Deployment)의 방법이 적용되었다. $\mathrm{QFD}$ 는 사용자 요구사항을 제품의 구성요소로 변환하는 방 법이다(Marsot, 2005). QFD의 절차는 먼저 고객 니즈를 포 함한 고객의 소리(VOC: Voice Of Customer)를 규명하고, $\mathrm{VOC}$ 를 충족하는 제품의 공학적 특성을 규명하여, 제품에 대 한 개발 목표를 설정하는 과정을 통하여 수행된다.

본 연구에서는 사용자 경험 측정을 통하여 이동리프트에 대한 사용자 요구사항들을 도출하였고, 이동리프트에 대한 구성요소를 Figure 1과 같이 Swivel bar, Boom, Mast, Base, Pendant, Actuator, Control box, Caster brake, Front caster, Emergency Lowering Device로 정의하였다.

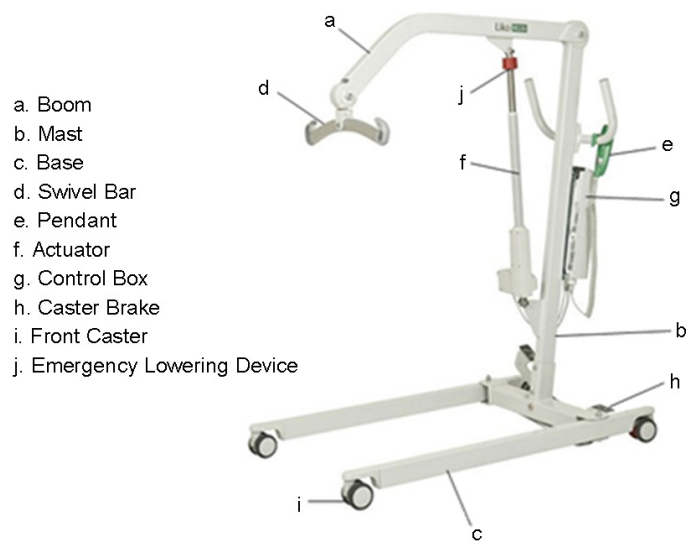

Figure 1. Components of mobile patient lift

마지막으로, 사용자 요구사항을 반영한 이동리프트의 디 자인 방향을 설정하기 위하여 품질의 집 $(\mathrm{HOQ}$; House of Quality)을 구하였다. 물론 이 과정에서 사용자 요구사항들 
의 상대적 중요도를 구하여야 하는데, 이를 위해 본 연구에 서는 분석계층과정(AHP; Analytic Hierarchy Process)을 활용하였고, 이동리프트 설계 전문가 1 인과 인간공학 전문가 1 인이 중요도 평가에 참여하였다. Table 4 는 분석계층과정 을 통하여 도출된 사용자 요구사항의 상대적 중요도이고, Figure 2는 이상의 과정을 통하여 도출된 품질의 집이다.

Table 4. Relative importance weight of requirements

\begin{tabular}{c|c}
\hline Users' requirements & Weight \\
\hline Easy lifting and lowering & 0.14 \\
\hline Easy movement & 0.15 \\
\hline Safe transferring & 0.19 \\
\hline Preventing caregiver's injuries & 0.13 \\
\hline Body stability while moving a patient & 0.11 \\
\hline Good compatibility with use environment & 0.06 \\
\hline Good interaction between patient \& sling & 0.09 \\
\hline Satisfactory shape & 0.07 \\
\hline Easy brake control & 0.06 \\
\hline
\end{tabular}

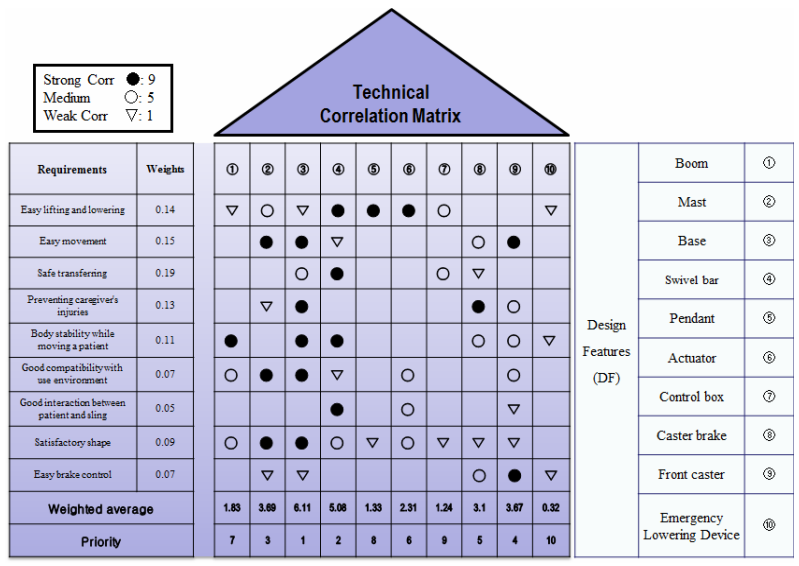

Figure 2. HOQ applied to mobile patient lift

분석 결과를 통하여, 본 연구에서는 사용자 요구사항을 반영한 이동리프트의 디자인 요소로 이동리프트의 Base, Swivel bar 등의 순서로 디자인의 중요성이 결정되었다. 이 동리프트의 Base는 환자를 이동하는 과정에서의 용이성과 밀접하게 관련되어 있어 사용자 경험 측정 결과를 체계적으 로 반영한 결과로 볼 수 있다. 또한, Swivel Bar는 환자에 대한 신체지지 기구 중 하나인 슬링 (sling) 과 밀접하게 관련 되어 있고, 리프트의 사용성 및 적합성에 큰 영향을 끼친다. 리프트 사용의 위험성은 대부분 슬링과 관련이 있다. 요양보
호사가 쉽게 사용할 수 있으며, 사용자가 슬링에 앉아 승, 하 강 시 편안하고 안락함을 느낄 수 있는 재질 및 형태를 갖추 어야 할 것이다. 또한 슬링의 고리는 사용자의 긁히지 않도 록 부드럽고, 내구성이 뛰어난 재료를 사용하여야 한다.

\section{User Experience Measurement for Four Wheeled Walker}

고령자 관련 사고 유형을 보면 알 수 있듯이 전체 사고 중 에서 낙상 관련 사고가 무려 절반 이상을 차지하고 있다. 낙 상을 방지할 수 있는 고령친화제품으로 가장 대표적인 것이 보행보조차가 있지만, 그에 대한 사용자 경험을 측정하고, 그 결과를 디자인에 반영함으로써 사용성의 불편과 안전성 을 높이고자 하는 노력은 이루어지지 못하였다. 본 연구에서 는 보행보조차를 사용한 경험이 있는 사용자들을 대상으로 여러 가지의 방법을 통하여 사용상의 경험을 측정하였고, 이 를 토대로 디자인 개선을 도출하였으며, 최종적으로 디자인 프로토타입을 개발하였다.

\subsection{Qualitative user experience measurement}

보행보조차의 정성적 사용자 경험을 측정하기 위하여 보 행보조차를 사용하는 고령자를 대상으로 인터뷰와 관찰 방 법이 활용되었다(Figure 3).
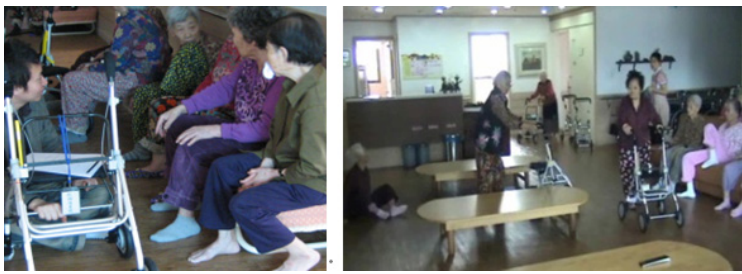

Figure 3. Interview and observation

인터뷰의 방법에서는 전화인터뷰와 심층인터뷰의 방법이 활용되었고, 관찰에서는 고령자가 보행보조차를 사용하는 과 정을 직접적으로 관찰하면서 사용자 경험을 측정하였다. 전 화인터뷰에서는 (주)삼주유니콘에서 판매된 보행보조차를 사용하고 있는 사용자 100 명을 대상으로 한 전화인터뷰와 광주광역시의 천혜경로원을 방문하여 22 명의 노인을 대상으 로 보행보조차의 사용 경험과 관련된 간략한 인터뷰와 사용 과정에 대한 관찰을 통하여 문제점을 발견하였다. 물론 사용 과정에 대한 장면을 캠코더로 기록하여 분석하였다. 
전화인터뷰에서는 사용 방법, 사용 목적, 사용해서 좋은 점, 불만족스러운 부분이나 개선할 점 등을 측정하였고, 심 층인터뷰에서는 의자와 가방 크기, 브레이크에 대한 사용, 손잡이 형태, 높이조절, 보행보조차 사용 시 피로를 느끼는 부분, 불편한 점 등에 대한 사용 경험을 측정하였다.

전화인터뷰 결과를 보면, 보행보조차 사용 목적에 대한 설 문(복수 응답)에 대해서는 허리 불편 때문이라고 답한 비 율이 62건, 다리 불편이라고 답한 비율이 37 건, 골다공증 2 건, 기타 의견이 5 건이 있었다(Figure 4 ).

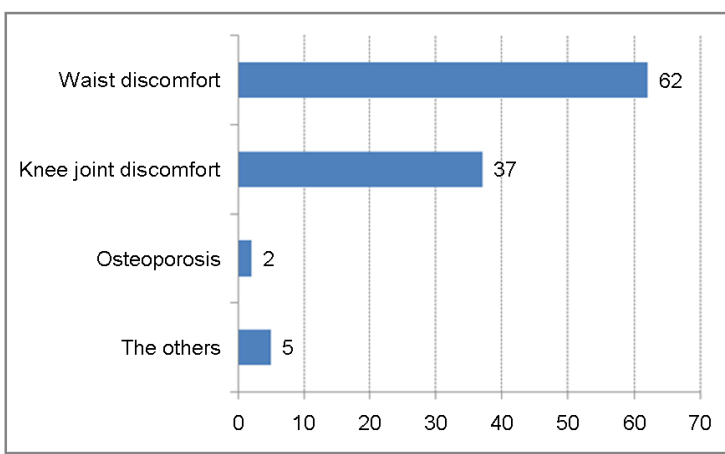

Figure 4. Reasons using walker

보행보조차에 대한 개선 사항에 대해서는 가방이 컸으면 좋겠다고 답한 인원이 5 명, 높낮이 조절이 쉬웠으면 좋겠 다고 답한 인원이 12 명, 사용 방법이 쉽게 이해되었으면 좋겠다고 답한 인원이 14 명, 손목에 무리가 가지 않았으면 좋겠다고 답한 인원이 5명, 의자가 조금 더 컸으면 좋겠다 고 답한 인원이 6 명, 가방색, 무게 등을 포함한 기타 의견 5 건, 그리고 특별한 니즈를 말하지 않은 인원이 53명이었다 (Figure 5).

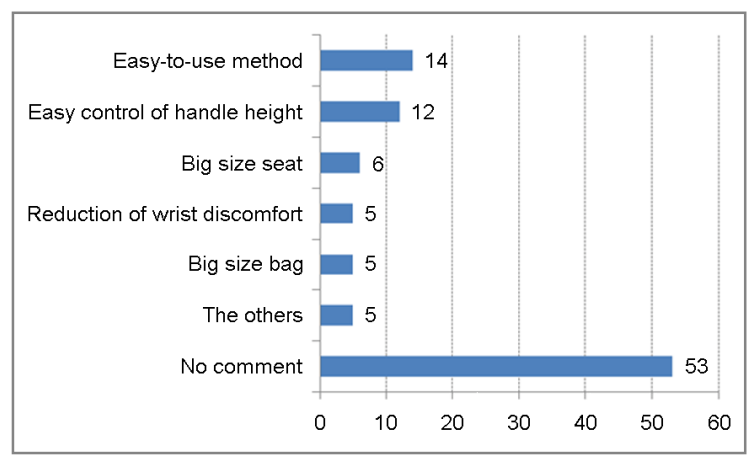

Figure 5. Needs for walker design
보행보조차를 사용한 이후에 좋아진 점을 묻는 설문에 대 하여, 허리를 펴고 걸을 수 있어 좋다고 답한 인원이 35명, 이동/걷기가 훨씬 쉬워졌다고 답한 인원이 38 명, 이동 중 쉴 수 있어 다리가 덜 아프다고 답한 인원이 17 명, 짐을 실을 수 있어 좋다고 답한 인원이 4 명, 기타 의견이 6 건이었다 (Figure 6).

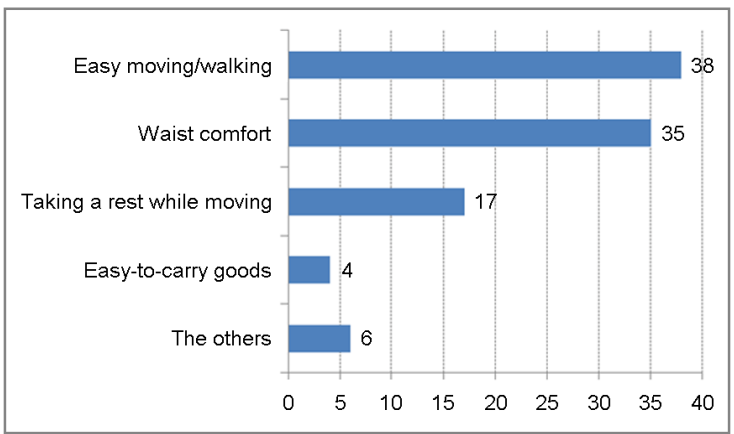

Figure 6. Good points while using walker

보행보조차 사용자에 대한 일대일 인터뷰를 통하여 측정 된 사용자 경험을 정리한 결과는 다음과 같다.

- 허리에 대한 통증을 제기한 분이 3 명

- 팔이나 팔뚝에 대한 통증을 제기한 분이 5 명

- 높이조절에 대한 불만족을 제기한 분이 2 명

- 의자에 대한 불편을 제기한 분이 2 명

•손목의 통증을 제기한 분이 3 명

- 브레이크 조작의 불편을 제기한 분이 2 명

- 제품의 무게에 대한 불만족을 제기한 분이 1 명

- 의견을 제기하지 않은 분이 3 명

또한 사용자 관찰을 통하여 도출된 사용상의 문제점들은 다음과 같다.

- 중심잡기가 힘들고 불안해 보인다.

- 브레이크 잡기가 힘들어 보인다.

- 엉덩이가 뒤로 많이 빠지면서 자세가 불안정해 보인다. 키가 크신 분들은 더욱 불안정한 자세를 취하게 된다.

- 손목이 많이 꺾여 불편해 보인다.

- 보행보조차의 방향전환이 힘들어 보인다.

\subsection{Four wheeled walker design considering user experience}

본 연구에서는 보행보조차의 사용자인 고령자들을 대상으 로 한 전화인터뷰와 일대일 인터뷰, 그리고 관찰을 통하여 
도출된 디자인과 관련한 사용자 경험 자료부터 다음과 같은 세 가지의 중요한 디자인 문제를 도출하였다.

첫째 허리, 다리, 손목 부분의 근골격과 관련된, 이것은 보 행보조차의 높이와 형태에 관련된 문제이다. 허리와 다리 부 분의 통증은 보행보조차 높이의 부적합성으로 인한 부적합 한 자세에 의하여 유발되는 것으로, 기본적으로는 보행보조 차의 높낮이에 대한 조절범위를 국내 고령자의 인체치수를 고려하여 디자인함으로써 고령 사용자가 자신의 치수에 적 합한 높이를 설정하여 사용하도록 유도하는 것이 필요하다. 또한 손목 부분의 통증은 보행보조차의 높이와도 관련있지 만, 손잡이의 형태와도 밀접한 관련이 있다. 손잡이 부분의 개선을 위해서는 손목관절의 ㄲㄲㄲ임이 최소화될 수 있는 디자 인 형태를 갖도록 디자인되어야 할 것이다.

둘째 보행보조차의 사용상의 불편함과 관련된 브레이크와 바퀴와 관련된 문제이다. 즉 브레이크 조작이 불편하고 좁은 공간에서의 방향전환 등을 용이하게 할 수 있는 디자인 방법 이 제공되는 것이 필요하다. 특히, 브레이크 조작의 어려움 은 손목 통증과도 관련되는 문제이다.

셋째 의자와 관련된 문제로, 이 부분은 의자 디자인에 있 어 고령 사용자들의 인체 크기, 특히 엉덩이 부분의 크기를 디자인에 반영하여야 할 것이다.

본 연구에서는 이상의 사용자 경험을 측정한 결과로부터 고령자가 잡기 편한 손잡이 형태, 방향전환이 용이한 바퀴, 그리고 고령자가 앉기에 적합한 의자 크기를 도출하였고, 그 결과를 반영하여 Figure 7 과 같이 보행보조차를 새롭게 디 자인하였다. 보행보조차를 사용할 때 사용자의 손목이 꺾이 지 않고, 브레이크 조작의 용이성을 높이며, 좁은 공간에서 의 방향전환이 용이할 수 있도록 하는데 중점을 두었다.

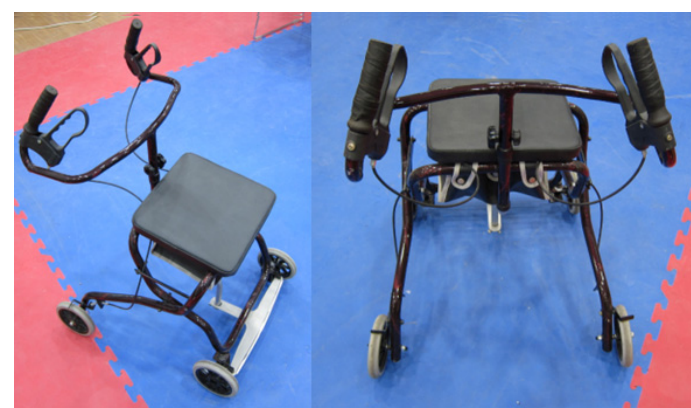

Figure 7. New Design Case of four-wheeled walker considering user experience

또한 보행보조차 사용과정에서의 신체적 불편함과 의자 사용의 편의성을 개선하기 위하여 우선적으로 보행보조차 사용자인 국내 65세 이상의 고령자 신체치수를 반영하였다.
그 결과는 Jung, et al.(2009)에서 연구되었던 결과를 반영 하였다(Table 5).

Table 5. Anthropometric design guidelines for walker

\begin{tabular}{c|c|c}
\hline Design part & Design factor & Dimension $(\mathrm{mm})$ \\
\hline \multirow{4}{*}{ Handle } & Girth & $61.5 \sim 105.5$ \\
\cline { 2 - 3 } & Width & Over 90.5 \\
\cline { 2 - 3 } & Height & $841 \sim 1074$ \\
\cline { 2 - 3 } & $\begin{array}{c}\text { Distance between hand grip } \\
\text { and brake handle }\end{array}$ & Below 94.5 \\
\cline { 2 - 3 } & Distance between hand grips & Below 382.5 \\
\hline \multirow{4}{*}{ Seat } & Height & Fixed type: 353.5 \\
\cline { 2 - 3 } & Width & Over 402 \\
\cline { 2 - 3 } Arm rest & Depth & Below 248 \\
\cline { 2 - 3 } & Distance between arm rests & Over 534.5 \\
\hline \multirow{4}{*}{} & Height &
\end{tabular}

\section{Conclusion}

본 연구에서는 고령친화제품의 사용자 경험 측정과 그 결 과를 디자인에 반영하는 방법에 대해 사례연구를 통하여 연 구하였다. 일반적으로 고령자의 이동은 일상생활을 위하여 가장 필수적인 요소인데, 비보행 고령자의 경우는 이동을 위 해 요양보호사의 도움이 필요하고, 이때 이동리프트의 사용 을 통하여 요양보호사의 신체적 부담을 줄여줄 수 있다. 또 한 보행 고령자의 경우에는 보행보조차의 사용을 통하여 안 정적이고 편리한 이동을 도움받을 수 있다.

그러한 측면에서 이동리프트와 보행보조차에 대한 사용자 들의 경험을 측정하고, 디자인 측면에서 개선되거나 중점적 으로 사용자의 특성을 반영하여야 할 요소들을 도출하거나 그 방법을 제시하였다.

특히 보행보조차의 경우에는 사용자 경험을 측정하고 그 결과를 반영한 디자인 사례를 제시하였다. 물론 본 연구는 사례연구의 관점에서 진행되었기 때문에 이론적 측면보다는 개념적 측면에서의 방법론과 실제적 진행 사례에 그 중요한 의미를 둘 수 있다. 


\section{Acknowledgement}

This study was supported by research fund from Korea University of Technology and Education during sabbatical year (2012).

\section{References}

Andreoni, G., Method for the analysis of posture and interface pressure of car drivers, Applied Ergonomics, Vol. 33, 511-522, 2002.

Chaffin, D.B. and Anderson, G.B.J, Occupational Biomechannics. New York: John Wiley \& Sons, 1991.

Fisk, A.D. and Roger, W.A., Handbook of Human Factors and the Older Adult, Academic Press, 1997.

Huang, H.C., et al., Assessing Risk of Falling in Older Adults. Public Health Nursing, Vol. 2, 2003.

Jung, K.T., et al., Anthropometric Analysis and Usability Evaluation of Four-wheeled Walker, J of the Ergonomics Society of Korea, Vol. 28, No. 2, 2009.

Kim, S., A case study on the repair and maintenance of elderly housing, Korea Institute of Construction Technology, 2004.

Korea Consumer Agency, Old People's Safety, 2007.

LF1050 Patient Lift Manual, LUMEX, GF Health Product, Inc., 2011.

Marsot, J., QFD: A methodological tool for integration of ergonomics at the design stage, Applied Ergonomics, 36, 185-192, 2005.

Milosavljevic, S., Influence of occupation on lumbar sagittal motion and posture, Ergonomics, 657-667, 2005.

Moller, J., Projected costs of fall related injury to older persons due to demographic, Change in Australia (Revised), 2003.

OSHA, Guidelines for Nursing Homes, U.S. Department of Labor, OSHA 3182, Occupational Safety and Health Administration, 2003.

Ryu, J. and Mun, M., A Novel Mobility Aid for Independent Daily Living of Elderly people, Journal of the Korean Society of Precision Engineering, Vol. 21, 2004.

Sadigh, S., et al., Falls and fall-related injuries among the elderly: a survey of residential-care facilities in a Swedish municipality, Journal of Community Health, Vol. 29, No. 2, 2004.

Simonton, K. and Wilcox, D., Frequently Asked Questions about Portable Total Body Patient/Resident Lifts, Department of Labor and Industries, 1999.
Tullis, T. and Albert, B., Measuring the user experience, Morgan *Kaufmann, 2008.

Waters, R., Product design issues related to safe patient handling technology, Human Factors and Ergonomics in Consumer Product Design: Uses and Applications, CRC Press, 2011.

Wikipedia, Patient Lift, http://en.wikipedia.org/wiki/Patient lift, 2012.

\section{Author listings}

\section{Kwang Tae Jung: ktjung@koreatech.ac.kr}

Highest degree: $\mathrm{PhD}$, Department of Industrial Engineering, KAIST Position title: Professor, Department of Industrial Design Engineering, Koreatech

Areas of interest: Applied Ergonomics and Design, Emotional Design, User Experience

Keyoung Jin Chun: chun@kitech.re.kr

Highest degree: $\mathrm{PhD}$, Department of Mechanical Engineering, Michigan State University

Position title: Chief Researcher, Smart Welfare Technology R\&D Group Areas of interest: Biomechanics, Senior Friendly Product Development

Byeong Hee Won: bhwon@kitech.re.kr

Highest degree: $\mathrm{PhD}$, Department of Mechanical Engineering, Hanyang University

Position title: Chief Researcher, Smart Welfare Technology R\&D Group Areas of interest: Biomechanics, Senior Friendly Product Development

Date Received : 2013-12-04

Date Revised :2013-12-08

Date Accepted : 2013-12-19 